\title{
Employee Stock Options: An Up-and-Out Protected Barrier Call
}

\author{
Chris K. Anderson, Cornell University \\ Neil Brisley, University of Western Ontario
}

A well-known numerical lattice model, widely used to value employee stock options (ESOs), can be interpreted as a variation on the up-and-out protected barrier call, a version of which is valued in closed form by Carr (1995). We clarify that valuation formula and extend it to take account of the reality of possible vesting date exercise by employees.

\section{Introduction}

Carr (1995) contributes a valuation formula for an up-and-out protected barrier call option. ${ }^{1}$ The up-and-out feature means that if the stock price reaches a fixed barrier value, (higher than the initial stock price) then the option is knocked out, and a rebate paid. It is protected for a fixed initial period of time during which it cannot be knocked out, whatever the stock price attained. In this paper we extend the model to make it applicable to the valuation of employee stock options (ESOs). We obtain a closedform solution that is potentially of significant practical use in its own right, and can serve as a benchmark against which to validate other more elaborate numerical models.

In the years since Carr's paper, the finance and accounting literature has sought ways to value ESOs, the single largest component of executive compensation. Valuation is necessary for financial reporting purposes and for economic decision-making, the ESO being valued from the firm's point of view, and this is the approach we take here. ${ }^{2}$ ESOs typically have a protection period of 1-4 years during which exercise is not permitted. At the end of the protection period, the ESO 'vests' and the employee is free to exercise the option at anytime until expiry, typically 10 years. In practice, the employee's early exercise decision may depend on idiosyncratic factors such as risk aversion and non-option wealth, and this makes the determination of the grant date value somewhat challenging. In an influential paper, Hull and White (2004) use a numerical lattice approach and assume that the employee exercises whenever the stock reaches a fixed multiple of the exercise price. This convenient assumption enables numerical estimates of the option value to be obtained. ${ }^{3}$ 
Since early exercise of an option effectively leads to a rebate, the Hull and White model has an up-and-out characteristic and, given the practical application, the benefits of a closed-form solution such as Carr's become apparent. To our knowledge, Carr's formula has attracted the interest of practitioners, yet it would appear to contain errors causing values to diverge from known rational bounds. We first present a corrected version of Carr's formula.

Next, we extend the corrected Carr formula to make it directly applicable to the valuation of ESOs. Carr's model assumes that a fixed dollar rebate is payable when the stock price achieves the barrier value. In reality and in the Hull and White lattice model, however, the inability of the employee to exercise the ESO before vesting implies that the stock price may attain values significantly higher than the barrier value at the vesting date, whereupon the employee's rebate is the full excess of stock price over exercise price. That is, the payoff at the vesting date can be strictly greater than the fixed rebate effectively available if the option is knocked out by reaching the barrier after the vesting date. We extend the corrected Carr formula to allow for this characteristic. Our extension is potentially of significant practical use in its own right, and as a benchmark against which to validate other more elaborate numerical models.

\section{Up-and-Out Protected Barrier Call Option}

We adopt the notation of Carr (1995). The option has exercise price $K$ and barrier $B$, which is above the initial stock price, $S_{0}$. The stock has dividend yield $\delta$, volatility $\sigma$, and the riskless rate is $r$. The option has expiration date $t_{2}$ and protection period $\left[0, t_{1}\right]$ during which it cannot be knocked out. At the end of this period, if the underlying stock price, $\mathrm{S}_{1}$, is at or above $B$, then the call is knocked out and a rebate, $R$, paid. Otherwise, the option remains alive. If, subsequently, the stock price hits the barrier, then the call is knocked out and the rebate paid. If the stock price does not hit the barrier before expiry, then it becomes a standard call, and is exercised if the stock price, $\delta_{2}$, is greater than $K$, and expires worthless otherwise.

Using risk-neutral valuation, our corrected version of Carr (1995, Proposition A, p. 175, Equation (2)) for the value of a European up-and-out call option with a protection period is 


$$
\begin{aligned}
& U_{0}= e^{-r_{1}} R N\left(\frac{-b+\mu t_{1}}{\sigma \sqrt{t_{1}}}\right)+R\left(\frac{B}{S_{0}}\right)^{(\mu-\tilde{\mu}) / \sigma^{2}}\left[G\left(t_{2}\right)-G\left(t_{1}\right)+H\left(t_{1}, t_{2}\right)\right] \\
&+e^{-\delta t_{2}} S_{0}\left[N_{2}\left(\frac{b-\hat{\mu} t_{1}}{\sigma \sqrt{t_{1}}}, \frac{b-\hat{\mu} t_{2}}{\sigma \sqrt{t_{2}}} ; \sqrt{\frac{t_{1}}{t_{2}}}\right)\right. \\
&-\exp \left(\frac{2 \hat{\mu} b}{\sigma^{2}}\right) N_{2}\left(\frac{b+\hat{\mu} t_{1}}{\sigma \sqrt{t_{1}}}, \frac{-b-\hat{\mu} t_{2}}{\sigma \sqrt{t_{2}}} ;-\sqrt{\frac{t_{1}}{t_{2}}}\right)-N_{2}\left(\frac{b-\hat{\mu} t_{1}}{\sigma \sqrt{t_{1}}}, \frac{k-\hat{\mu} t_{2}}{\sigma \sqrt{t_{2}}} ; \sqrt{\frac{t_{1}}{t_{2}}}\right) \\
&\left.+\exp \left(\frac{2 \hat{\mu} b}{\sigma^{2}}\right) N_{2}\left(\frac{b+\hat{\mu} t_{1}}{\sigma \sqrt{t_{1}}}, \frac{k-2 b-\hat{\mu} t_{2}}{\sigma \sqrt{t_{2}}} ;-\sqrt{\frac{t_{1}}{t_{2}}}\right)\right] \\
&- e^{-r t_{2}} K\left[N_{2}\left(\frac{b-\mu t_{1}}{\sigma \sqrt{t_{1}}}, \frac{b-\mu t_{2}}{\sigma \sqrt{t_{2}}} ; \sqrt{\frac{t_{1}}{t_{2}}}\right)\right. \\
&-\exp \left(\frac{2 \mu b}{\sigma^{2}}\right) N_{2}\left(\frac{b+\mu t_{1}}{\sigma \sqrt{t_{1}}}, \frac{-b-\mu t_{2}}{\sigma \sqrt{t_{2}}} ;-\sqrt{\frac{t_{1}}{t_{2}}}\right) \\
&-N_{2}\left(\frac{b-\mu t_{1}}{\sigma \sqrt{t_{1}}}, \frac{k-\mu t_{2}}{\sigma \sqrt{t_{2}}} ; \sqrt{\frac{t_{1}}{t_{2}}}\right) \\
&\left.+\exp \left(\frac{2 \mu b}{\sigma^{2}}\right) N_{2}\left(\frac{b+\mu t_{1}}{\sigma \sqrt{t_{1}}}, \frac{k-2 b-\mu t_{2}}{\sigma \sqrt{t_{2}}} ;-\sqrt{\frac{t_{1}}{t_{2}}}\right)\right],
\end{aligned}
$$

where

$$
\begin{aligned}
& G(t)=N\left(\frac{-b+\tilde{\mu} t}{\sigma \sqrt{t}}\right)+\exp \left(\frac{2 \tilde{\mu} b}{\sigma^{2}}\right) N\left(\frac{-b-\tilde{\mu} t}{\sigma \sqrt{t}}\right), \\
& H\left(t_{1}, t_{2}\right)=N_{2}\left(\frac{-b+\tilde{\mu} t_{1}}{\sigma \sqrt{t_{1}}}, \frac{b-\tilde{\mu} t_{2}}{\sigma \sqrt{t_{2}}} ;-\sqrt{\frac{t_{1}}{t_{2}}}\right) \\
& +\exp \left(\frac{2 \tilde{\mu} b}{\sigma^{2}}\right) N_{2}\left(\frac{-b-\tilde{\mu} t_{1}}{\sigma \sqrt{t_{1}}}, \frac{b+\tilde{\mu} t_{2}}{\sigma \sqrt{t_{2}}} ;-\sqrt{\frac{t_{1}}{t_{2}}}\right), \\
& \mu \equiv r-\delta-\frac{\sigma^{2}}{2}, \\
& \tilde{\mu} \equiv \sqrt{\mu^{2}+2 \sigma^{2} r}, \\
& \hat{\mu} \equiv \mu+\sigma^{2}=r-\delta+\frac{\sigma^{2}}{2}, \\
& b \equiv \ln \left(\frac{B}{S_{0}}\right),
\end{aligned}
$$

and where 


$$
N(x) \equiv \int_{-\infty}^{\pi} \frac{\mathrm{e}^{-z^{2} / 2}}{\sqrt{2 \pi}} d z
$$

denotes the standard (univariate) normal distribution function and

$$
N_{2}\left(x_{1}, x_{2} ; \rho\right)=\int_{-\infty}^{x_{1}} \int_{-\infty}^{x_{2}} \frac{1}{2 \pi \sqrt{(1-\rho)^{2}}} \exp \left\{\frac{1}{2(1-\rho)^{2}}\left[z_{1}^{2}-2 \rho z_{1} z_{2}+z_{2}^{2}\right]\right\} d z_{2} d z_{1}
$$

denotes the standard bivariate normal distribution function. ${ }^{4}$

\section{Extension: Full Exercise-Value Rebate at Vesting Date Exercise}

For an employee stock option, consider the Hull and White (2004) assumption that the employee has a strict policy of exercising options when the stock reaches a barrier, $B>K$. At times $t>t_{1}$, this will occur when $S_{t}=B$ and a rebate, $R=B-K$, is payable. However, at the vesting date, $t=t_{1}$, the stock price can already have surpassed $B$, whereupon a larger rebate, $S_{1}-K$, is payable. We now extend the corrected Carr formula to allow for this characteristic.

The conditional expected value of vesting date payoffs (when those payoffs are strictly restricted to $\mathrm{R}$ ) is expressed in our corrected Carr equation by the first term

$$
e^{-r t_{1}} R N\left(\frac{-b+\mu t_{1}}{\sigma \sqrt{t_{1}}}\right)
$$

Conditional on the stock reaching the barrier at vesting, we now permit the vesting date payoff to be $\mathrm{S}_{1}$ $-K$, whereupon its conditional expected value is

$$
e^{-r t_{1}} N\left(\frac{-b+\mu t_{1}}{\sigma \sqrt{t_{1}}}\right)\left[S_{0} \exp \left(r-\delta-\sigma \frac{\phi(\alpha)}{1-N(\alpha)}\right)-K\right]
$$

where $\propto=[b-) r-\delta)] / \sigma$, and $\emptyset(\propto)$ is the standard normal pdf, $\emptyset(\propto)=\left(\frac{1}{\sqrt{2 \pi}}\right) e^{-\alpha^{2} / 2}$. The expected stock price conditional on hitting the barrier is simply the initial stock price multiplied by the natural exponent raised to the mean of a truncated normal random variable. 


\section{Illustrative Example}

Figure 1 illustrates barrier option values as a function of the barrier B, with parameters $S_{0}=1, K=1, \delta=0, \sigma=60 \%, t_{1}=3$ years, $t_{2}=10$ years and $r=10 \%$. The Black-Scholes value of the corresponding standard European call is $\$ 0.806147$. Intuitively, as the barrier B increases, the barrier option value should converge to the Black-Scholes value. The original Carr (1995) formulation approximates our corrected formula for smaller values of B, but eventually becomes decreasing in B, contrary to our intuition for this option. Our corrected formula and our extended formula (with augmented potential payoffs at vesting date exercise) each approach the Black-Scholes value as B increases - when $B=20$, both formulae are within $1 \%$ of the Black-Scholes value, and when $B=100$ the values are within $0.06 \%$ of Black-Scholes. Further, our extended formula has a strictly higher value than the corrected formula, due entirely to the augmented potential payoffs at the vesting date.

As further verification of our analytical results, we perform Monte Carlo simulation to obtain numerical estimates of the value of the basic up-and-out protected barrier call, and the extended version (with augmented potential payoffs at vesting date). Using 3000 time steps and 10,000 replications we obtain numerical estimates (for $B=2,3,4, \ldots, 20$ ) that differ from our analytical valuations by a maximum absolute deviation of $0.595 \%$ and an average absolute deviation of $0.273 \%$.

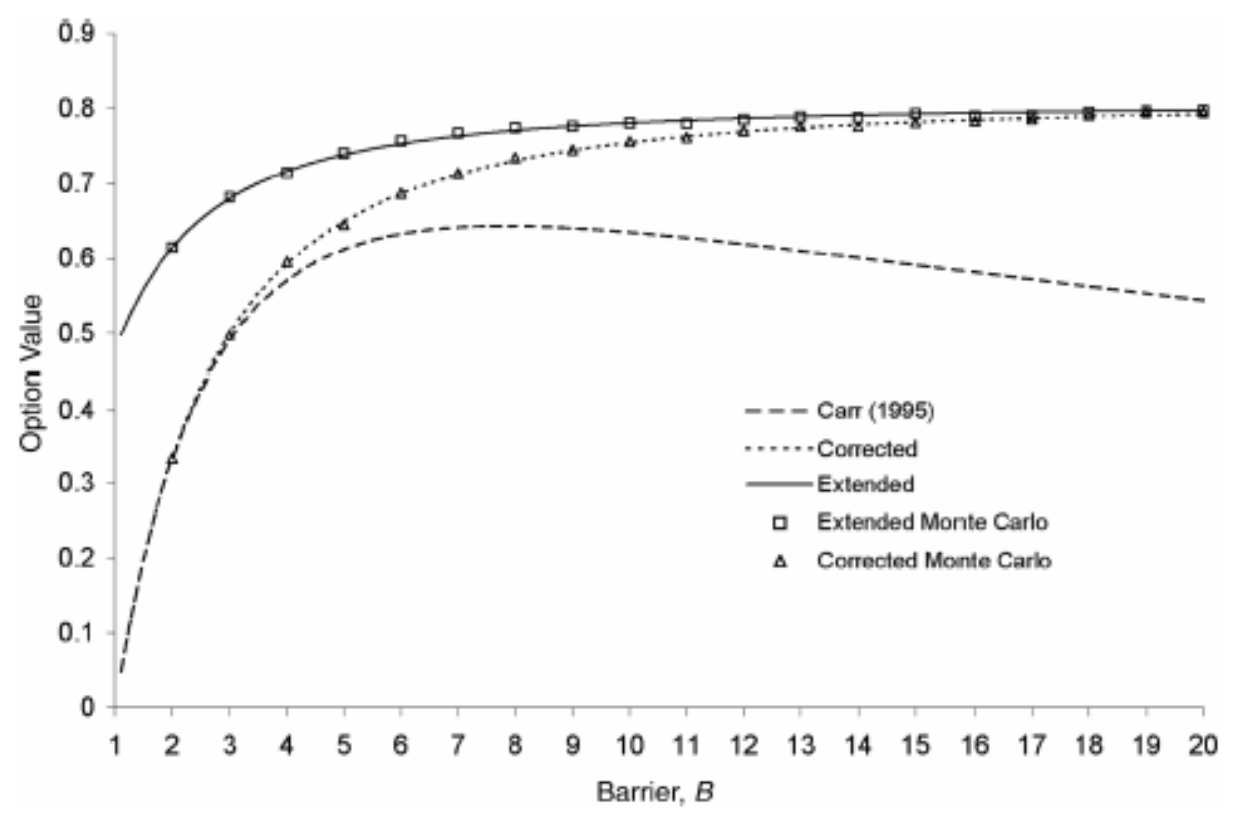

Figure 1. Barrier option values as a function of the barrier $B$. 


\section{Acknowledgements}

Brisley acknowledges support from Social Sciences and Humanities Research Council of Canada standard research grant \#410-2007-1564.

\section{References}

Carr, P. (1995) Two extensions to barrier option valuation. Applied Mathematical Finance, 2(3), pp. 173209.

Henderson, V. (2005) The impact of the market portfolio on the valuation, incentives and optimality of executive stock options. Quantitative Finance, 5(1), pp. 35-47.

Heynen, R. and Kat, H. (1994) Partial barrier options. Journal of Financial Engineering, 3(3), pp. 253-273.

Hull, J. and White, A. (2004) How to value employee stock options. Financial Analysts Journal, 60(1), pp. 114-119.

\footnotetext{
${ }^{1}$ Heynen and Kat (1994) address the valuation of barrier options when the barrier is not active over the option's entire life.

${ }^{2}$ Henderson (2005) is one of several papers to examine ESO valuation and incentives from the employee's point of view and contains a discussion and further references.

${ }^{3}$ Financial Accounting Standards Board Statement FAS123R makes compulsory the expensing of all ESOs in the US, effective for fiscal years commencing after 15 June 2005. Similar rules have been implemented by the International Accounting Standards Board. FAS123R permits several methodologies but specifically cites the Hull and White (2004) model and illustrates its use with numerical examples, as does Securities Exchange Commission Staff Accounting Bulletin \#107.
}

4 The error originates from the original proof in Appendix A3 (p. 200) of Carr (1995). There, a probability decomposition is written

$$
P\left(X_{2}<k_{2}, \tau>t_{2}, X_{1} \leq b\right)=P\left(M_{01} \leq b, X_{2}<k_{2}, \tau>t_{2}, X_{1} \leq b\right)+P\left(M_{01} \leq b, X_{2}<k_{2}, \tau>t_{2}, X_{1} \leq b\right) .
$$

However, the right-hand side should read

$=P\left(M_{01} \leq b, X_{2}<k_{2}, \tau>t_{2}, X_{1} \leq b\right)+P\left(M_{01}>b, X_{2}<k_{2}, \tau>t_{2}, X_{1} \leq b\right)$.

As a result the third line,

$$
=P\left(M_{01} \leq b, X_{2}<k_{2}\right)+P\left(M_{01} \leq b, X_{2}<k_{2}, \tau>t_{2}, X_{1} \leq b\right),
$$

should read

$$
=P\left(M_{01} \leq b, X_{2}<k_{2}\right)+P\left(M_{01}>b, X_{2}<k_{2}, \tau>t_{2}, X_{1} \leq b\right) .
$$

Equation (A3) should end

$$
=N_{2}\left(\frac{b-\mu t_{1}}{\sigma \sqrt{t_{1}}}, \frac{k_{2}-\mu t_{2}}{\sigma \sqrt{t_{2}}} ; \sqrt{\frac{t_{1}}{t_{2}}}\right)-\exp \left(\frac{2 \mu b}{\sigma^{2}}\right) N_{2}\left(\frac{b+\mu t_{1}}{\sigma \sqrt{t_{1}}}, \frac{k_{2}-2 b-\mu t_{2}}{\sigma \sqrt{t_{2}}} ;-\sqrt{\frac{t_{1}}{t_{2}}}\right)
$$


Which results in (A4) becoming

$$
P\left(\tau>t_{2}, X_{1} \leq b\right)=N_{2}\left(\frac{b-\mu t_{1}}{\sigma \sqrt{t_{1}}}, \frac{b-\mu t_{2}}{\sigma \sqrt{t_{2}}} ; \sqrt{\frac{t_{1}}{t_{2}}}\right)-\exp \left(\frac{2 \mu b}{\sigma^{2}}\right) N_{2}\left(\frac{b+\mu t_{1}}{\sigma \sqrt{t_{1}}}, \frac{-b-\mu t_{2}}{\sigma \sqrt{t_{2}}} ;-\sqrt{\frac{t_{1}}{t_{2}}}\right),
$$

Resulting in

$$
\begin{aligned}
P\left(X_{2}>k_{2}, \tau>t_{2}, X_{1} \leq b\right)= & P\left(\tau>t_{2}, X_{1} \leq b\right)-P\left(X_{2} \leq k_{2}, \tau>t_{2}, X_{1} \leq b\right) \\
= & N_{2}\left(\frac{b-\mu t_{1}}{\sigma \sqrt{t_{1}}}, \frac{b-\mu t_{2}}{\sigma \sqrt{t_{2}}} ; \sqrt{\frac{t_{1}}{t_{2}}}\right)-\exp \left(\frac{2 \mu b}{\sigma^{2}}\right) \\
& \times N_{2}\left(\frac{b+\mu t_{1}}{\sigma \sqrt{t_{1}}}, \frac{-b-\mu t_{2}}{\sigma \sqrt{t_{2}}} ;-\sqrt{\frac{t_{1}}{t_{2}}}\right) \\
& -N_{2}\left(\frac{b-\mu t_{1}}{\sigma \sqrt{t_{1}}}, \frac{k_{2}-\mu t_{2}}{\sigma \sqrt{t_{2}}} ; \sqrt{\frac{t_{1}}{t_{2}}}\right)+\exp \left(\frac{2 \mu b}{\sigma^{2}}\right) \\
& \times N_{2}\left(\frac{b+\mu t_{1}}{\sigma \sqrt{t_{1}}}, \frac{k_{2}-2 b-\mu t_{2}}{\sigma \sqrt{t_{2}}} ;-\sqrt{\frac{t_{1}}{t_{2}}}\right) .
\end{aligned}
$$

